\title{
EXAMINING NEUROLOGICAL AND NEURODEGENERATIVE DISORDERS RELATED TO AGING AND ELDERLY
}

Elcin Huseyn, Research Laboratory of Intelligent Control and Decision-Making Systems in Industry and Economics, Azerbaijan State Oil and Industry University, Baku, Azerbaijan, ORCID ID: https://orcid.org/0000-0001-5965-7419

\section{DOI: https://doi.org/10.31435/rsglobal_conf/30042021/7529}

Abstract. Aging is a complex bio-psycho-social process. The elderly population has increased in the world and in our country in recent years. It is predicted that the ratio of the elderly population to the general population in our country will increase over the years. In old age, the incidence of neurodegenerative diseases and stroke increases. It is clear that these diseases cause a serious care and treatment burden for the society and patient relatives. It is increasingly important to answer whether a noticeable reduction in cognition of the elderly is a normal consequence of old age or an early sign of a neurodegenerative disease such as Alzheimer's disease. This importance will be better understood if the efforts and progress made worldwide to improve the early diagnosis and treatment of neurodegenerative diseases are considered. The purpose of this review is to summarize the effects of old age and senile on the nervous system, to identify diseases with a marked increase in incidence in old age and to evaluate these diseases from a general point of view.

Keywords: Aging, senility, dementia, Alzheimer's disease, Parkinson's disease, stroke

Aging. Aging is a complex bio-psycho-social process, and it is species specific, not organ and cell. The average and maximum life expectancy of each species is determined. Aging refers to a process in which the physiological spare capacity of the creature gradually decreases after the maturation process is completed, and its life functions are disrupted accordingly. Aging is a lifelong process starting from the thirties. Although the old age limit is generally accepted as 65 years, today biological age is considered more important than chronological age and chronological age is not accepted as an absolute indication in the evaluation of treatment and survival (1).

- Epidemiology of Old Age

According to the data of the Turkish Statistical Institute (TUIK), the life expectancy for Turkey (respectively total; male and female) was 73.9 in 2010; 71.6; While it was 76.3 , it was 75.4 in 2020; 72.7; It is estimated to be 78.3 years (http://www.tuik.gov.tr; 08.07.2010). 488,568 people aged 85 and over live in Turkey. It is thought that the elderly population ratio will reach $9.8 \%$ of the entire population in 2025, and this value corresponds to 8.253 .000 elderly people.

- Aging and Changes in Nervous System

With aging, atherosclerosis predisposition occurs in the brain. Oxidative damage, vascular endothelial dysfunction, and inflammatory response cause vascular changes. Cerebral blood flow is reduced by up to $20 \%$. These changes cause an increased risk of stroke. In addition, the decrease in brain perfusion may cause a decrease in cognitive functions.

With aging, lipofuscin accumulation, gliosis and neuronal degeneration are observed in neurons in the brain. Glial cell reactivation occurs with retraction and expansion, synapse loss and reorganization in dendrites with the accumulation of insoluble proteins such as tau, amyloid and alpha synuclein in the extracellular space. In this process, in which a decrease in brain volume occurs, the decrease in brain cells and volume is tried to be compensated by using new synaptic connections (2).

This decrease in brain volume accelerates from the $60 \mathrm{~s}$. With age, changes in neurotransmitter systems may occur without cell damage. A marked decrease in monoaminergic and dopaminergic transmission is observed. This situation increases the susceptibility of the elderly to depression and extrapyramidal side effects. With aging, changes occur in the neuroendocrine system.

There is an increase in glucocorticoid levels. This increase predisposes to hippocampal neuron damage, ischemia, and exotoxicity. This contributes to an increase in the incidence of stroke and Alzheimer's disease (AD). 
Normal cognitive changes associated with aging; It is characterized by a moderate decrease in memory, planning and processing speed in particular. A decrease is also observed in learning performance (3). However, this decrease is not significant enough to affect daily life. Impairment of knowledge, vocabulary, communication and perception is not expected in the elderly without dementia (4).

- Aging and Neurological Diseases

With aging, there is a significant increase in the incidence of some neurological diseases. Neurodegenerative diseases and stroke are the leading diseases. Age is the most important risk factor for $\mathrm{AD}$, and the prevalence of the disease increases exponentially every 5 years after the age of 65 . In a study conducted in Turkey, the prevalence of $\mathrm{AD}$ over the age of 70 was found to be $10 \%(5)$. The average age of onset of Parkinson's disease (PD) is between 50-60 years old, and the prevalence increases with age. The prevalence of many neurodegenerative diseases such as multi system atrophy (MSA), progressive supranuclear palsy (PSP), Lewy body dementia (LCD), frontotemporal dementia (FTD), amyotrophic lateral sclerosis (ALS) increases with age.

Age is also one of the most important risk factors for stroke (6). $70 \%$ of those who have a stroke are over the age of 65 . The incidence of stroke increases 2-fold for each decade after the age of 55. The two periods with a high incidence of epilepsy are the first year of life and after 60 years of age. The higher frequency of metastatic brain tumors, cerebrovascular events (CVE) and degenerative brain diseases in the elderly contributes to the increased incidence of epilepsy. Obstructive sleep apnea is common in people of middle age and older. Although myasthenia gravis (MG) is mostly known as a young disease, $M G$ is most frequently bimodal in women between the ages of 20-30 or over 50, and most frequently in men over the age of 50. In this review, dementia, $\mathrm{PH}$, and stroke, which increase in frequency with aging, will be emphasized.

Dementia. The term dementia was formed by adding a negative suffix to the word "mens", which means mind in Latin, and is used to mean the loss of acquired mental functions. On the basis of generally accepted concepts today, dementia is a permanent and often progressive condition in terms of its natural course, as a result of damage to the central nervous system (CNS), blurring of consciousness, impairment of more than one cognitive area without delirium, and accordingly, daily life activities are affected and cannot be maintained at the old level. a clinical pictur.

Although many different causes have been defined in the etiology of dementia, AD, which is the most common cause of dementia, accounts for more than two-thirds of all dementias. As the population ages, the incidence of $\mathrm{AD}$ and the burden of the disease gradually increases. With the development of new treatments, recognition of early signs of AD and other dementia subtypes will become increasingly important.

\section{- General Approach to Dementia Clinic}

It is difficult to detect and evaluate dementia in daily practice. In a study, it was shown that the diagnosis of dementia was missed in $21 \%$ of the patients, and $20 \%$ of the patients diagnosed with dementia did not have dementia (7). Many patients with dementia do not complain about forgetfulness, moreover, while the complaints of forgetfulness reported by individuals are generally not related to dementia, it has been shown that the relatives reporting that the person has memory problems has a higher correlation with the diagnosis of dementia (8). However, relatives may be delayed in recognizing dementia symptoms and may perceive these symptoms as a normal consequence of old age. Dementia patients often have problems in more than one cognitive domain. They may encounter problems and difficulties in learning new information, performing complex tasks, establishing cause-effect relationships, spatial skills, language functions and behaviors. Dementia also needs to be differentiated from delirium and depression. Dementia tends to start insidiously, while delirium begins acute or subacute. Sometimes depression can be confused with dementia. Psychomotor slowing may be observed in patients with depression and their effort in cognitive tests is reduced. Dementia patients strive to be successful in tests, but they fail.

Dementia is often due to more than one cause, especially as the process progresses. In addition, comorbid illnesses and some medications can cause poor cognition in patients with dementia. Cerebrovascular disease is also present in a significant number of patients with dementia. While most of the information required for the diagnosis of dementia can be obtained with reliable close-up information and bedside tests, diagnosis of vascular dementia (VaD) continues to be difficult despite additional information obtained from imaging methods. AD, LCD, FTD, VaD and Parkinson's dementia (PD) constitute the major neurodegenerative dementia syndromes. In non-neurodegenerative dementias, there may be no progression, improvement can be observed, and some can be treated. 
The symptomatology of dementia syndrome can be classified into three main categories: cognitive, behavioral, and functional (Table 1). In addition, signs and symptoms of motor, autonomic and sleep disorders are important in the diagnosis and differential diagnosis of some dementia syndromes.

Table 1. Questioning cardinal and secondary areas in demas (ADL: Activities of daily living)

\begin{tabular}{|c|c|c|}
\hline \multirow{7}{*}{ Cognitive } & Memory & $\begin{array}{l}\text { Recent: Personal and current events of the recent past; } \\
\text { Far: Primary school teacher, the schools he attended, } \\
\text { marriage, retirement dates, etc. }\end{array}$ \\
\hline & Attention & Fluctuation, concentration, susceptibility \\
\hline & Tongue & $\begin{array}{l}\text { Word finding, comprehension, reading, writing, } \\
\text { calculation difficulties }\end{array}$ \\
\hline & Visual-spatial functions & $\begin{array}{l}\text { Being able to navigate in foreign / familiar spaces, } \\
\text { changes in the typeface (orthographic) }\end{array}$ \\
\hline & Executive functions & Problem solving, judgment, abstraction disorders \\
\hline & Praxis & Difficulties in using tools, dressing, sitting and walking \\
\hline & Gnosis & Recognizing objects, separating them in space \\
\hline \multirow{4}{*}{ Behavioral } & Personality changes & Apathy, disinhibition, social inappropriateness \\
\hline & Mood disorders & $\begin{array}{l}\text { Grief, unwillingness, restlessness, restlessness, irritability, } \\
\text { inappropriate joy, not leaving after spouse }\end{array}$ \\
\hline & Perception disorders & Visual and other hallucinations \\
\hline & Thinking disorders & Theft, infidelity, Capgras, and other delusions \\
\hline \multirow{3}{*}{ Functional } & GYAs on the street & Work, travel, finances, shopping, social relations \\
\hline & GYAs at home & $\begin{array}{l}\text { Hobbies, using household appliances, cooking, other } \\
\text { household chores, minor repairs, newspaper-TV attention }\end{array}$ \\
\hline & Self care & $\begin{array}{l}\text { Eating, bathing, dressing, make-up, shaving, toilet } \\
\text { mechanics, sphincter control }\end{array}$ \\
\hline Engine & \multicolumn{2}{|c|}{$\begin{array}{l}\text { Gait disturbance, falls, freezing, imbalance, slowness of movement, } \\
\text { weakness, melting, twitching }\end{array}$} \\
\hline Autonomous & \multirow{2}{*}{\multicolumn{2}{|c|}{$\begin{array}{l}\text { Incontinence, impotence, orthostatism, constipation, sweating } \\
\text { ehavior disorder, excessive daytime sleepiness, sleep apnea syndrome }\end{array}$}} \\
\hline Sleep & & \\
\hline
\end{tabular}

The most frequently affected area in the cognitive area is memory. The patient repeats the same issues, asks the same questions over and over, loses his personal belongings. They can forget the food on the stove and confuse their appointments. The deterioration of visual spatial functions begins with the loss of way in lesser known spaces, and patients may confuse the places of their rooms at home in later periods. It starts with deterioration in language function, narrowing of vocabulary and naming difficulty and progresses to aphasia, and speech may disappear completely in the advanced stage. Apractic disorders with difficulties in the use of simple current devices (comb, scissors, etc.), and gnostic disorders in the form of inability to recognize objects and faces can be seen. Executive dysfunction results in a loss of abstraction, planning, reasoning, and mental flexibility. Suitable solutions cannot be produced for the problems encountered in daily life (9).

Behavioral symptoms include loss of spontaneity (apathy), impulse control disorders (inappropriate behavior, hypersexuality, hyperphagia). There may be thought and perception disorders. There may be delusions of theft, infidelity, and abandonment. Visual hallucinations are more common, especially at night. Agitation, purposeless repetitive movements may be observed. Depression is common and can be overlooked due to dementia, so it should be evaluated specifically.

While functional symptoms are limited to some situations such as traveling to an unknown place and financial difficulties in the early stage, assistance may be required even in self-care activities such as hygiene and nutrition in the advanced stage. When evaluating motor disorders, attention should be paid to extrapyramidal symptoms such as walking with small steps, bradykinesia, and tremor. It should be kept in mind that signs of paresis may be encountered in $\mathrm{VaD}$ and motor neuron disease may accompany FTD.

- Approach to the Diagnosis of Dementia

The first focus on a patient with suspected dementia diagnosis is the detailed history taken from the patient and the people who know the patient well. Changes in the patient's cognitive 
functions and behavior over time should be questioned. A 30-minute examination time will probably not be sufficient for a full dementia examination. Neurological, physical and cognitive examinations of the patient, biochemistry examinations and imaging will be required. Detailed neuropsychological tests may be required for some patients. It should also be questioned whether the patient takes medication (anticolonergic, sedative, hypnotic, antipsychotic, narcotic analgesic) that will affect his cognition. According to DSM-V diagnostic criteria, the term "major neurocognitive disorder" was used instead of dementia. For the diagnosis of major neurocognitive disorder according to DSM-V; Without the delirium picture and psychiatric illness to explain the current cognitive impairment, the person should have functional loss according to the previous mental state, daily living activities (ADL) should be affected and there should be a significant impairment in at least one cognitive area. DSM-V diagnostic criteria for major neurocognitive impairment.

As a result of the history and clinical evaluations, there should be a significant effect in at least one of the following cognitive areas.

\section{Learning and Memory \\ Language \\ Executive functions \\ Complex attention \\ Perceptual-motor functions \\ Behavior- social cognition}

The affliction should include a significant loss relative to the person's previous functional state. Cognitive impairment should be so severe that daily living activities are carried out independently from others. If associated with a neurodegenerative disease such as Alzheimer's, mental impairment must be insidious and progressive. Cognitive impairment should not have occurred during delirium. Cognitive impairment should not be better explained by another neuropsychiatric disease.

In a meta-analysis in which the Mini Mental Test (MMT) and other dementia screening tests were evaluated, the sensitivity of the screening tests was found to be $75-92 \%$ and the specificity $81 \%$ $91 \%$ (10). While evaluating these tests, the education level and normal cognitive level of the patient should be taken into consideration. MMT maximum score is 30 points and below 24 points is considered significant for dementia. MMT is significantly affected by training. In a community study, mean MMT scores were found 26 for people with 5-8 years of education and 22 for people with 4 years or less (11). The Mini-Cog test is a screening test in which the clock drawing test and 3 word recall are performed together. The sensitivity and specificity of this test are similar to MMT. It was found to be correlated with the clock drawing test and MMT scores (12). Clinical dementia rating scale (CDR) is used for staging AD. Different neuropsychiatric test batteries have been developed to evaluate different cognitive areas. Among these tests, appropriate ones should be selected according to the clinical picture of the patient. Among these batteries, tests evaluating memory function are considered to be more useful for the diagnosis of dementia. Animal naming test, Modified Boston Naming test, MMT, word list learning tests can be used to distinguish cognitive changes associated with $\mathrm{AD}$ and normal aging.

The American Academy of Neurology (AAN) recommends screening for B12 deficiency and hypothyroidism in patients with dementia. In patients with rapidly progressive and early onset dementia, performing cerebrospinal fluid (CSF) examinations and serological tests, and evaluating neuroimaging and EEG examinations may be useful for diagnosis. Routine genetic evaluation is not recommended for AD. It is uncertain that $\mathrm{AD}$ will develop even in homozygous apolipoprotein $\mathrm{E} 4$ carriers (13). This assessment can be confusing because of the high frequency of false positive and false negative results.

It is recommended to perform non-contrast computed tomography $(\mathrm{CT})$ or magnetic resonance imaging (MRI) examinations for every patient with dementia (14). Imaging is particularly important in rapidly progressing dementias with early onset ( $<60$ years) focal neurological findings. Hippocampal atrophy is evident in AD. Hippocampal atrophy is associated with cognitive impairment. Dementia patients with atherosclerotic risk factors may have silent cerebrovascular disease. After detecting these patients with neuroimaging methods, a more aggressive antiatherosclerotic treatment can be applied. Positron emission tomography (PET) has been used frequently in both daily practice and research in recent years in patients with dementia. Differential diagnosis of different dementia syndromes can be made with fluorodeoxyglucose-PET (FDG-PET) and supportive findings can be obtained for the diagnosis of dementia. With amyloid PET, important information can be obtained in terms of progression to $\mathrm{AD}$ in people with mild cognitive impairment. 
The point that has been emphasized recently in the diagnosis of dementia is the increase in tau and phospho-tau levels in the CSF and the decrease in $\beta$-amyloid42 levels, which are the most advanced methods accepted in the diagnosis of AD with their high sensitivity and specificity (15). In addition, these markers are thought to be important in the conversion of patients with mild cognitive impairment to AD.

Stroke. According to the definition of the World Health Organization, stroke; It is a clinical syndrome characterized by the rapid settlement of signs and symptoms of focal loss of cerebral function without any apparent cause other than vascular causes. Stroke; It includes different subtypes such as brain infarction, intracerebral hemorrhage (ICH) and subarachnoid hemorrhage (SAH). Cerebral infarction is seen in $80 \%$ (70-85\%), ICH in 15\% (7-15\%) and SAH in 5\% (2-8\%) of all strokes.

Stroke is the third most common cause of death in the world and the most common cause of disability in the adult society. The incidence of stroke increases significantly in the elderly population and reaches 4 per thousand in people over the age of 45 (16). It is clear that prevention and treatment of stroke is and will be very important in our country, where the elderly population is increasing significantly. In Turkey, SVR is the second most common cause of death with $15 \%$ and the second most common cause of DALY (Year of Life Adapted to Loss of Function) (17).

Age is the most important unalterable risk factor for ischemic stroke and spontaneous ICH. The incidence of stroke increases twice for every decade after the age of 55. Hypertension is the strongest modifiable risk factor for ischemic stroke and ICH.

In the prevention of ischemic stroke, strict control of atherosclerotic risk factors, appropriate treatment of asymptomatic carotid stenosis and atrial fibrillation, avoiding the use of oral contraceptives in women with migraine and hypercoagulability, treatment of severe sleep apnea, proper diet and exercise are important. Five subgroups are defined according to the "causative classification system for ischemic stroke" (CCS) classification used in the classification of ischemic stroke. These are large artery, atherosclerosis, cardioaortic embolism, small vessel occlusion, other causes, and unexplained stroke. Large and small vessel atherosclerosis and cardioaortic embolism increase with age.

Stroke is a disease that requires rapid diagnosis and rapid intervention. It is recommended that patients with sudden onset of neurological deterioration and lateralizing findings should be evaluated rapidly in the emergency room and neurological imaging should be performed after their vital signs are stable. After the stroke subtype is determined, treatment should be started rapidly and the patient should be followed up with close monitoring. Stroke recurrence and death from stroke can be significantly reduced with optimal medical therapy. In our country, stroke units and stroke centers are established for rapid and effective intervention in stroke, and thrombolytic therapy and intravascular interventions are applied in these centers. Considering the increasing incidence of stroke, it is clear that the number and quality of these centers will need to be increased.

Parkinson's disease. Parkinson's disease is the most common condition among the movement disorder group diseases after essential tremor. Parkinson's Disease is typically a disease of middle and old age, with an average age of 60 (40-70 years), and both incidence and prevalence increase with age. In this respect, Parkinson's Disease can be defined as a disease associated with aging.

Although Parkinson's Disease has traditionally been considered as a motor system disease, it is now accepted as a much more complex disease with a motor and nonmotor (autonomic, behavioral, cognitive and sensory) involvement pattern. Eosinophilic cytoplasmic inclusions (Lewy bodies) and dopaminergic neuron loss in substantia nigra are seen as pathological findings. Cardinal clinical symptoms of Parkinson's Disease are bradykinesia, rigidity, tremor and postural instability. The disease often begins asymmetrically in one half of the body and the most common initial symptom is resting tremor in one extremity. The gold standard in the diagnosis of Parkinson's Disease is neurological examination. The diagnosis of Parkinson's Disease is made on the basis of history and clinical findings and excluding other possible diagnoses. REM sleep behavior disorder, anosmia and constipation, which are also accepted as premotor findings of Parkinson's Disease, are seen in most patients. These findings can occur years before the onset of motor symptoms. When examined with appropriate mental tests, mental dysfunctions ranging from mild to severe are detected in most of the patients. Particularly in elderly patients and in the later years of the disease, PD may develop in some of the patients, this rate is $15-20$. It can reach very high figures of $50-80 \%$ in years (18).

Parkinson's Disease typically starts insidiously and progresses slowly over the years. With dopaminergic treatment, the prognosis of the patients improved significantly and the life expectancy was prolonged. With the progression of the disease, the clinical picture is aggravated by the motor complications associated with dopaminergic treatment, decreased response to dopaminergic treatment, 
and exacerbation of nondopaminergic symptoms. In the advanced stage, motor involvement symptoms such as generalized bradykinesia, postural instability and freezing, bulbar involvement and dementia determine the prognosis.

Multisystem atrophy. Multiple system atrophy (MSA) is a sporadic and rapidly progressive neurodegenerative disease that occurs in adulthood (mean age of onset: 54.2). Findings seen in MSA are parkinsonism, cerebellar, pyramidal findings and autonomic involvement. It is clinically characterized by two different motor involvement. The type with more pronounced parkinsonism findings is named as the type MSA-P and the type with the cerebellar findings more pronounced as MSA-C. Inclusion bodies stained with alpha synuclein are observed in its pathology and therefore MSA is classified as a synucleinopathy.

Corticobasal degeneration. In corticobasal degeneration, other movement disorders such as asymmetric akinetic, rigid parkinsonism and accompanying dystonia, myoclonia, and cortical symptoms with apraxia and cortical sensory loss are observed. Foreign hand phenomenon can be seen. Average onset $(63 \pm 7.7)$ is in the later stages of life.

Amyotrophic lateral sclerosis. Amyotrophic lateral sclerosis (ALS) is characterized by the loss of motor neurons in the primary motor cortex, brainstem, and medulla spinalis. It generally starts at an advanced age. The average age of onset is between 60 and 65 years old. It is a progressive and fatal disease and the average survival is 2.5-3.5 years. Coexistence of upper and lower motor neuron findings is typical. History and neurological examination are essential in diagnosis. Imaging and electrophysiological examinations can be used for differential diagnosis.

\section{REFERENCES}

1. Karan M. Akif, (2013). Aging and the Elderly, Neurology Basic Book. 2nd Edition P. 1517

2. Ropper AH, Brown RJ, Ropper A (2009) Morphologic and physilogic changes in aging nervous system. Principler of Neurology, 9th ed. McGraw Hill, Boston, P. 580

3. Small, S. A., Stern, Y., Tang, M., \& Mayeux, R. (1999). Selective decline in memory function among healthy elderly. Neurology, 52 (7), 1392-1392.

4. Vandenberge R, Tournoy J, (2005). Cognitive aging and Alzheimer's disease. Postgrad Med J, 81: 343-52

5. Gurvit, H., Emre, M., Tinaz, S., Bilgic, B., Hanagasi, H., Sahin, H., ... \& Harmanci, H. ( 2008). The prevalence of dementia in an urban Turkish population. American journal of Alzheimer's disease and other dementias, 23 (1), 67-76.

6. Gurvit H., Bilgic B. (2013) Neurology Basic Book 2nd Edition P. 938

7. Barrett, J. J., Haley, W. E., Harrell, L. E., \& Powers, R. E. (1997). Knowledge about Alzheimer disease among primary care physicians, psychologists, nurses, and social workers. Alzheimer's Disease \& Associated Disorders, 11 (2), 99-106.

8. Carr, D. B., Gray, S., Baty, J., \& Morris, J. C. (2000). The value of informant versus individual's complaints of memory impairment in early dementia. Neurology, 55 (11), 1724-1727.

9. Gurvit İ. H. (2011). Dementia Syndrome, Alzheimer's Disease and Non-Alzheimer's Dementias. Istanbul Medical Faculty Neurology Book, p: 443

10. Tsoi, K. K., Chan, J. Y., Hirai, H. W., Wong, S. Y., \& Kwok, T. C. (2015). Cognitive tests to detect dementia: a systematic review and meta-analysis. JAMA internal medicine, 175 (9), 1450-1458.

11. Crum, R. M., Anthony, J. C., Bassett, S. S., \& Folstein, M. F. (1993). Population-based norms for the MiniMental State Examination by age and educational level. Jama, 269 (18), 2386-2391.

12. Royall, D. R., Cordes, J. A., \& Polk, M. (1998). CLOX: an executive clock drawing task. Journal of Neurology, Neurosurgery \& Psychiatry, 64 (5), 588-594.

13. Farrer, L. A., Brin, M. F., Elsas, L., Goate, A., Kennedy, J., Mayeux, R., ... \& Risch, N. J. (1995). Statement on use of apolipoprotein E testing for Alzheimer disease. JAMA, 274 (20), 1627-1629.

14. Knopman, D. S., DeKosky, S. T., Cummings, J. L., Chui, H., Corey-Bloom, J., Relkin, N., ... \& Stevens, J. C. (2001). Practice parameter: Diagnosis of dementia (an evidence-based review) Report of the Quality Standards Subcommittee of the American Academy of Neurology. Neurology, 56 (9), 1143-1153.

15. Humpel, C. (2011). Identifying and validating biomarkers for Alzheimer's disease. Trends in biotechnology, 29 (1), 26-32.

16. Shepherd O. (2011). Definitions, Classification, Epidemiology and Risk Factors in Cerebrovascular Diseases. Istanbul Medical Faculty Neurology Book, p: 235

17. Ünüvar, N., Mollahaliloğlu, S., \& Help, N. (2006). Turkey Burden of Disease Study, Ankara: TC Ministry of Health.

18. Emre M, Hanağası H, Şahin Hüseyin A, Yazıcı J. (Year) Movement Disorders, Istanbul Medical Faculty Neurology Book p: 517 